Archives of Clinical and Medical Case Reports

doi: $10.26502 /$ acmcr.96550038

Volume 2, Issue 5

Case Report

\title{
A Case of Impending Cardiac Tamponade Caused by Effusive Constrictive Pericarditis
}

\author{
Catalina Sanchez-Alvarez ${ }^{1}$, Monia Werlang ${ }^{1}$, Oludamilola Oluleye ${ }^{2}$, Mohamad H Yamani ${ }^{2 *}$ \\ ${ }^{1}$ Departments of Internal Medicine, Mayo Clinic, Jacksonville, Florida, United States \\ ${ }^{2}$ Cardiovascular Medicine, Mayo Clinic, Jacksonville, Florida, United States
}

*Corresponding Author: Dr. Mohamad H Yamani, Cardiovascular Medicine, Mayo Clinic, Davis 7E, 4500 San

Pablo Rd, Jacksonville, Florida 32224, United States, Tel: 904-953-7279; Fax: 904-953-2911; E-mail: yamani.mohamad@mayo.edu

Received: 12 October 2018; Accepted: 19 October 2018; Published: 23 October 2018

\begin{abstract}
Pericarditis is the most common pericardial disease worldwide, and can be recurrent in one third of the patients with viral and idiopathic pericarditis [1]. The diagnosis of pericarditis should be based on clinical criteria, history, clinical findings, electrocardiographic (ECG) changes and evidence of a pericardial effusion [2]. Elevation of the inflammatory markers and evidence of pericardial inflammation by an imaging technique such as contrast enhancement on the pericardium-CT scan or pericardial edema and pericardial late gadolinium enhancement on cardiac magnetic resonance imaging (MRI) are additional supportive criteria in the diagnosis [1].
\end{abstract}

Keywords: Cardiac Tamponade; Pericarditis; Pericardium-CT scan; Magnetic resonance imaging

\section{Case Study}

A 33 year old man, with history of recurrent pericarditis, presented with a new episode of chest pain, associated with EKG changes suggestive of pericarditis (Figure 1). A cardiac MRI showed normal thickness of the pericardium with trace effusion (Figure 2). There was no delayed gadolinium enhancement of the pericardium. Patient had extensive workup that was positive for Coxsackie B infection. Patient was started on aspirin and colchicine. A week later, patient presented to the emergency department with worsening symptoms of shortness of breath. On physical 
examination, blood pressure 90/66 mm hg, pulse 90/min, elevated jugular venous pressure with y descent, pulsus paradoxus, pulsatile hepatomegaly, and mild peripheral edema. Laboratory testing showed ESR of $36 \mathrm{~mm} / \mathrm{hr}$, and a CRP of $156.6 \mathrm{mg} / \mathrm{L}$. Chest X-ray showed enlargement of the cardiac silhouette (Figure 3). A high resolution CT scan of the chest showed moderate size pericardial effusion (Figure 4) that was not present before in the initial MRI. A transthoracic echocardiography (TTE) showed evidence of a large circumferential pericardial effusion (Figure 5) with inferior vena cava (IVC) plethora (Figure 6) and both right atrium (RA) and right ventricle (RV) collapse consistent with impending cardiac tamponade. An urgent pericardiocentesis was performed and drained a one liter of sero-sanguineous pericardial fluid with WBC: 1403; PMN: 44\%, Lymph: 45\%. Cultures were negative and cytology was negative for malignancy. A follow up TTE evidenced resolution of the pericardial effusion, and diffuse thickening of the pericardium, with persistence of a mild respiratory variation in the left ventricle (LV) and right ventricle (RV) filling concerning for pericardial constriction (Figure 7). A heart catheterization confirmed ventricular intra-dependence on simultaneous measurements of the LV and RV consistent with pericardial constriction. Patient was diagnosed with an effusive constrictive pericarditis, and was started on a trial of steroids following which he had significant clinical improvement.

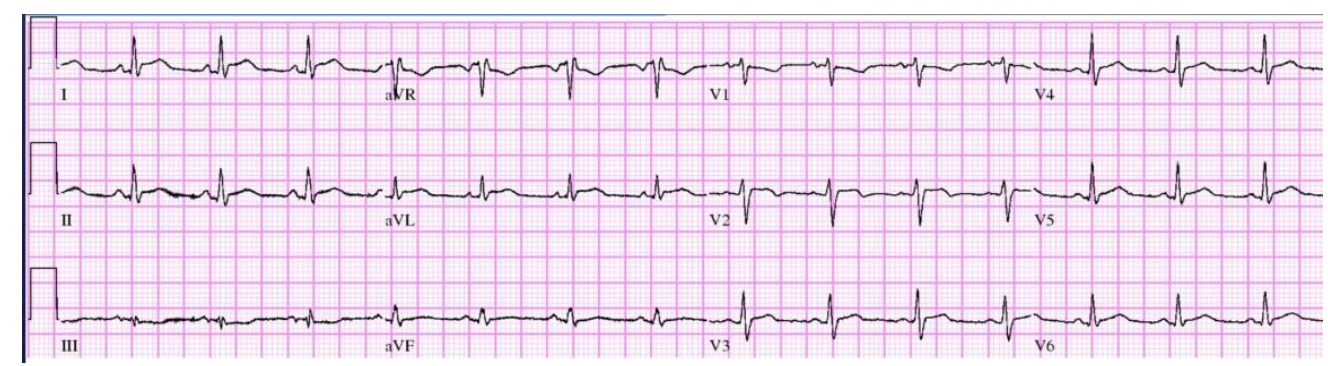

Figure 1: ECG.

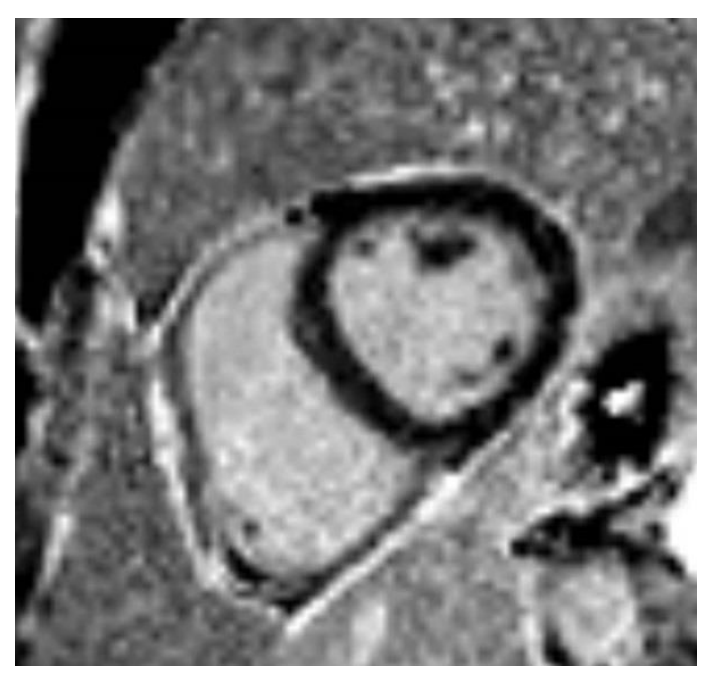

Figure 2: Cardiac MRI. 


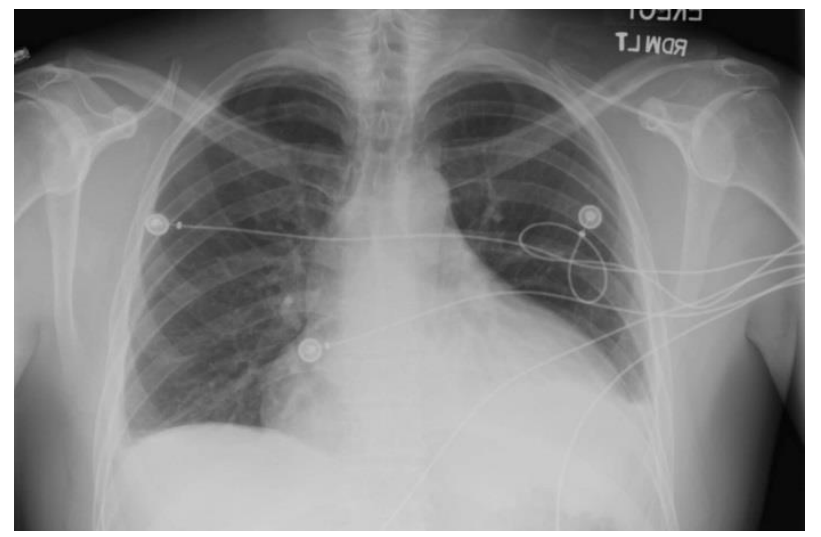

Figure 3: Chest X-ray.

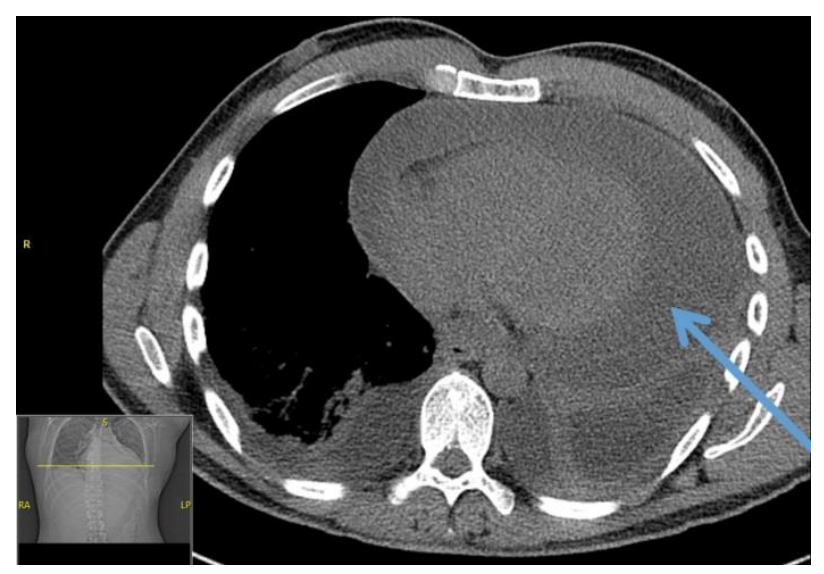

Figure 4: Chest CT scan.

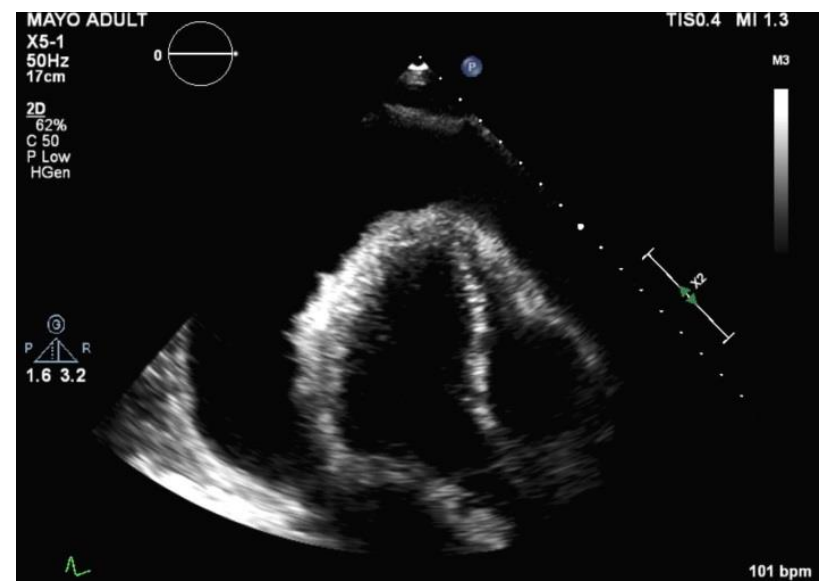

Figure 5: Transthoracic echocardiography. 


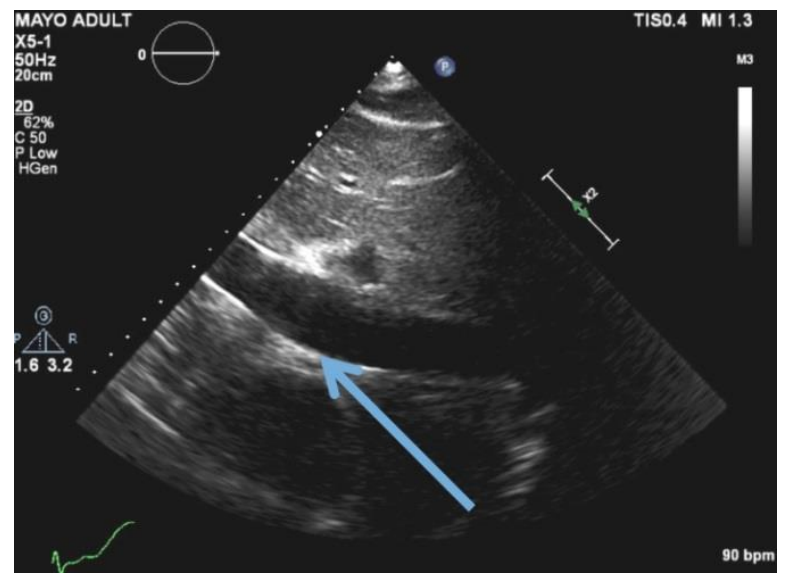

Figure 6: Inferior Vena cava.

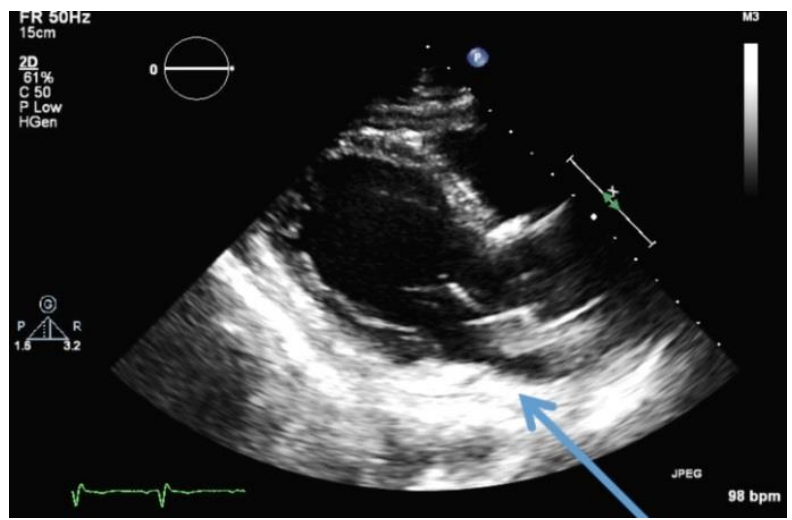

Figure 7: Transthoracic echocardiography post pericardiocentesis.

\section{Discussion}

Cardiac imaging is frequently used in the diagnosis of pericardial disease. The multimodal integration of different imaging techniques is needed in certain cases [3, 4]. TTE should be used in all the cases of acute pericarditis to identify effusions, and to evaluate for hemodynamic compromise as a reflection of cardiac tamponade [5, 6]. Other diagnostic approaches: hemodynamic cardiac catheterization may be necessary in establishing the diagnosis of constrictive pericarditis when the echocardiogram findings are not conclusive [5]. MRI has become the gold standard for the evaluation of the pericardium. The advantages include: lack of ionizing radiation, better anatomic evaluation and providing physiologic information regarding the cardiac function and wall motion in a non-invasive manner [7]. The sensitivity for late gadolinium enhancement has been reported to range from 94-100\% [8, 9]. Even highly sensitive modalities may initially be noncontributory in the initial phase of the disease. Particularly in our case the evidence of a normal MRI could have been due to an early stage of the disorder. And sometimes multiple imaging techniques are used in association with clinical findings to provide a confident diagnosis [8]. Although an important number of patients will require pericardiectomy, some cases have a predominantly inflammatory and reversible pericardial reaction, so a trial of anti-inflammatory agents should be considered before advocating pericardiectomy [10]. 


\section{References}

1. Imazio M, Gaita F, LeWinter M. Evaluation and Treatment of Pericarditis: A Systematic Review. JAMA 314 (2015): 1498-1506.

2. Khandaker MH, Espinosa RE, Nishimura RA, et al. Pericardial Disease: Diagnosis and Management. Mayo Clin Proc 85 (2010): 572-593.

3. Verhaert D, Gabriel RS, Johnston D, et al. The Role of Multimodality Imaging in the Management of Pericardial Disease. Circ Cardiovasc Imaging 3 (2010): 333-343.

4. Cosyns B, Plein S, Nihoyanopoulos P, et al. European Association of Cardiovascular Imaging (EACVI) position paper: Multimodality imaging in pericardial disease. Eur Heart J Cardiovasc Imaging 16 (2015): $12-31$.

5. Klein AL, Abbara S, Agler DA, et al. American Society of Echocardiography clinical recommendations for multimodality cardiovascular imaging of patients with pericardial disease: endorsed by the Society for Cardiovascular Magnetic Resonance and Society of Cardiovascular Computed Tomography. J Am Soc Echocardiogr off Publ Am Soc Echocardiogr 26 (2013): 965-1012.

6. Imazio M, Trinchero R. Myopericarditis: Etiology, management, and prognosis. Int J Cardiol 127 (2008): $17-26$.

7. Groves R, Chan D, Zagurovskaya M, et al. MR Imaging Evaluation of Pericardial Constriction. Magn Reson Imaging Clin 23 (2015): 81-87.

8. Young PM, Glockner JF, Williamson EE, et al. MR imaging findings in 76 consecutive surgically proven cases of pericardial disease with CT and pathologic correlation. Int J Cardiovasc Imaging 28 (2012): 10991109.

9. Taylor AM, Dymarkowski S, Verbeken EK, et al. Detection of pericardial inflammation with late enhancement cardiac magnetic resonance imaging: initial results. Eur Radiol 16 (2006): 569-574.

10. Syed F, Ntsekhe M, Mayosi B, et al. Effusive-constrictive pericarditis. Heart Failure Reviews 18 (2012): 277-287.

Citation: Catalina Sanchez-Alvarez, Monia Werlang, Oludamilola Oluleye, Mohamad H Yamani. A Case of Impending Cardiac Tamponade Caused by Effusive Constrictive Pericarditis. Archives of Clinical and Medical Case Reports 2 (2018): 157-161.

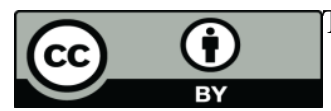

This article is an open access article distributed under the terms and conditions of the

Creative Commons Attribution (CC-BY) license 4.0 